\title{
Un caso de trombosis venosa parcial del segmento terminal de la vena mesentérica superior en un paciente con hepatopatía crónica
}

\author{
A case of partial venous thrombosis of the terminal segment of the superior mesenteric vein in a patient with \\ chronic liver disease \\ Pág. 2-9 \\ Recibida: 23/09/19 \\ M.D José Vega Molina* Gastón Vega Salazar, Estudiante de Medicina** \\ ${ }^{*}$ ) Director Médico Centro Médico TAC Alajuela S.A, Radiología General Neuroradiología, Costa Rica. \\ Aprobada:26/09/19 \\ $\left.{ }^{* *}\right)$ Estudiante de Medicina Universidad de Ciencias Médicas (UCIMED), San José, Costa Rica.
}

\section{Resumen}

Introducción: La isquemia intestinal tiene diversas causas y presentaciones que se pueden clasificar como agudas o crónicas. También se subdividen en causas arteriales, venosas y no oclusivas. La oclusión venosa mesentérica predomina en aproximadamente el $10 \%$ de los casos de isquemia intestinal. Las trombosis venosas están muy relacionadas a problemas de coagulabilidad, así como otras enfermedades como síndrome de anticuerpos antifosfolípidos, policitemia vera, síndrome de hepatopatía crónica o incluso al uso de anticonceptivos o al estado de hipercoagulabilidad en embarazos.

Caso: Paciente masculino de 52 años que se presenta con una hepatopatía crónica e hipertensión portal, hepatoesplenomegalia y con dolor abdominal insidioso que lo hace consultar con un especialista gastroenterólogo, el cual le solicita realizarse un ultrasonido abdominal. El paciente se presenta en ayunas y en el examen se le encuentra un hígado con aumento de la ecogenicidad, con superficie hepática micronodular, sin nódulos ni masas, sin dilatación de vías biliares intra o extrahepáticas y con vena porta y venas suprahepáticas permeables con calibre y aspecto normal teniendo al doppler color flujo normal. Vesícula biliar sin litiasis. Páncreas con morfología y con aspecto normal sin embargo inferior y medial a la cabeza pancreática se aprecia un área hipoecoica tubular, heterogénea y de bordes irregulares.

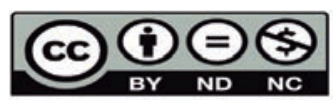

Discusión: La circulación venosa también se puede ver comprometida en la estrangulación del intestino como lo observado en volvulus, intusucepción o en obstrucción en asa.

La trombosis de la vena mesentérica aguda más comúnmente ocurre cuando existen condiciones patológicas como estados de hipercoagubilidad o neoplasias proliferativas causando trombosis de ramas venosas pequeñas con la consecuente reducción drástica del retorno venoso y formación de vasos colaterales. Varios métodos de imágenes están disponibles para su diagnóstico actualmente, cada cual tiene sus ventajas y desventajas. Entre estos está el ultrasonido convencional de alta resolución, ultrasonido doppler color, tomografía axial computada multicorte realizada con medio de contraste I.V, los cuales fueron utilizados en este caso. También se puede utilizar como gran aporte Resonancia Magnética (angioresonancia con gadolonio), que en este caso no se utilizó, ya que estaba claro el diagnóstico y por limitaciones económicas. Como otra opción, se puede realizar una angiografía digital del sistema arterial y venoso mesentérico, pero este procedimiento está reservado para casos de diagnóstico complicado con las técnicas no invasivas.

Conclusión: Por la información revisada y el catastrófico pronóstico de la enfermedad se deben hacer los mayores esfuerzos para pensar en esta entidad en pacientes con dolor abdominal y poseedores de factores de riesgo y así explotar más las posibilidades diagnósticas que pueden brindar la Tomografía Axial Computada, el ultrasonido convencional y el ultrasonido Doppler a color. 
Palabras Claves: Trombosis venosa mesentérica, Trombosis parcial vena mesentérica, Trombosis, Vena Mesentérica, Hepatopatía

A case of partial venous thrombosis of the terminal segment of the superior mesenteric vein in a patient with chronic hepatopathy

Background: Intestinal ischemia has various causes and presentations that can be classified as acute or chronic. They are also subdivided into arterial, venous and non-occlusive causes. Mesenteric venous occlusion predominates in approximately $10 \%$ of intestinal ischemia cases. Venous thrombosis are closely related to coagulability problems, as well as other diseases such as antiphospholipid antibody syndrome, polycythemia vera, portal hypertension, or even the use of contraceptives or the state of hypercoagulability in pregnancies.

Case Presentation: A 52-year-old male patient who presents a chronic hepatopathy with portal hypertension, hepatosplenomegaly and with insidious abdominal pain that makes him consult with a gastroenterologist, whom suggests for an abdominal ultrasound. The patient was on a fasting state for examination purposes, in which an increased echogenictity was found in the liver, with a micronodular surface, no nodules or masses, no dilation of intra or extrahepatic bile ducts and with a permeable portal and suprahepatic veins with normal size and appearance. Color-doppler showed a normal flow and thickness. Biliary vesicle without lithiasis. Pancreas with normal morphology and appearance, however inferior and medial to the pancreatic head, there is a hypoechoic tubular, heterogeneous area with irregular edges.

Discussion: The venous circulation can also be compromised on intestine strangulation as observed in volvulus, intussuception or obstruction in the intestinal loop. Acute mesenteric vein thrombosis most commonly occurs when there are pathological conditions such as hypercoagubility states or proliferative neoplasms causing thrombosis of small venous branches with the consequent drastic reduction of venous return and collateral vessel formation. Several imaging methods are currently available for diagnosis, each has its advantages and disadvantages. Among these the conventional high resolution ultrasound, color-doppler ultrasound, multislice computed axial tomography performed with I.V contrast media, which were used in this case can be mentioned. It can also be used as a great contribution the MRI (angiosonance with gadolonium), which in this case was not used, since the diagnosis was clear and due to economic limitations. As another option, a digital angiography of the mesenteric arterial and venous system can be performed, but this procedure is reserved for cases of complicated diagnosis with non-invasive techniques.

Conclusion: Due to the revised information and the catastrophic prognosis of the disease, the greatest efforts should be made to think of this entity in patients with abdominal pain and risk factors and thus exploit further the diagnostic possibilities that can be provided by computed axial tomography, conventional ultrasound and color-doppler ultrasound.

Key words: Mesenteric venous thrombosis, Mesenteric vein partial thrombosis, Thrombosis, Mesenteric vein, Hepatopathy.

\section{Introducción}

La isquemia intestinal tiene diversas causas y presentaciones $^{1,2}$. La Isquemia mesentérica es clasificada en dos formas; aguda y crónica y es subdividida por causas arteriales, venosas y no oclusivas. Con respecto a la clínica el criterio general es que presenta síntomas clínicos no especificos8. La oclusión venosa mesentérica se presenta en aproximadamente un $10 \%$ de los casos de isquemia intestinal ${ }^{3}$.

La trombosis venosa ocurre en pacientes con síndrome de hipergoagubilidad como enfermedad de células falciformes, síndrome de anticuerpos antifosfolípidos, policitemia vera, deficiencia de 
Seguidamente se le realiza un ultrasonido doppler a color del eje esplenoportal detectándose que el segmento hipoecoico de la vena mesentérica estaba parcialmente permeable con señal color y espectro venoso de la vena. (Fig .2)

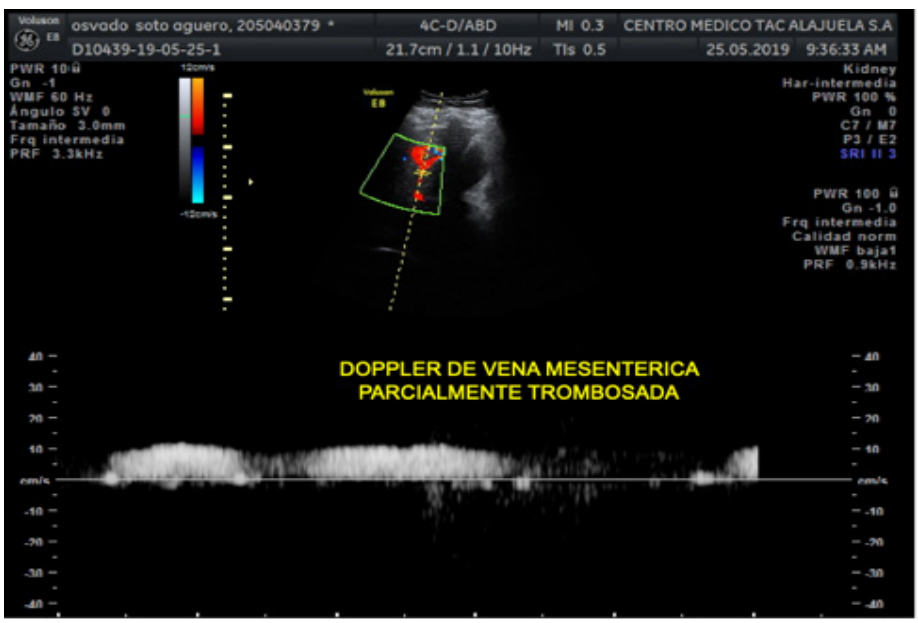

Fig 2. Se le realiza doppler color al segmento venoso trombosado y se aprecia que está estenosando parcialmente el segmento terminal de la vena mesentérica superior y presenta flujo sanguíneo en área anterior del lumen venoso lo que indica que está parcialmente estenosada.

Al realizarse posteriormente el examen tomográfico se demostró tanto en los reformateados en plano axial, coronal como sagital (Fig 3, Fig 4, Fig 5 ) que hay un área hipodensa en el segmento terminal de la vena mesentérica superior compatible con una trombosis venosa parcial de la vena mesentérica superior en su segmento terminal la cual está ensanchada $21 \mathrm{~mm}$ que mide $17 \mathrm{~mm}$ de ancho con un defecto de llenado posterolateromedial por trombo mural de $9,8 \mathrm{~mm}$ de espesor que se extiende en un segmento de unos $5 \mathrm{~cm}$. Se encontró también aspecto normal de la vena porta, permeablidad normal de la vena esplénica y tortuosidad de la misma sin trombosis, esplenomegalia y los hallazgos de hepatopatía

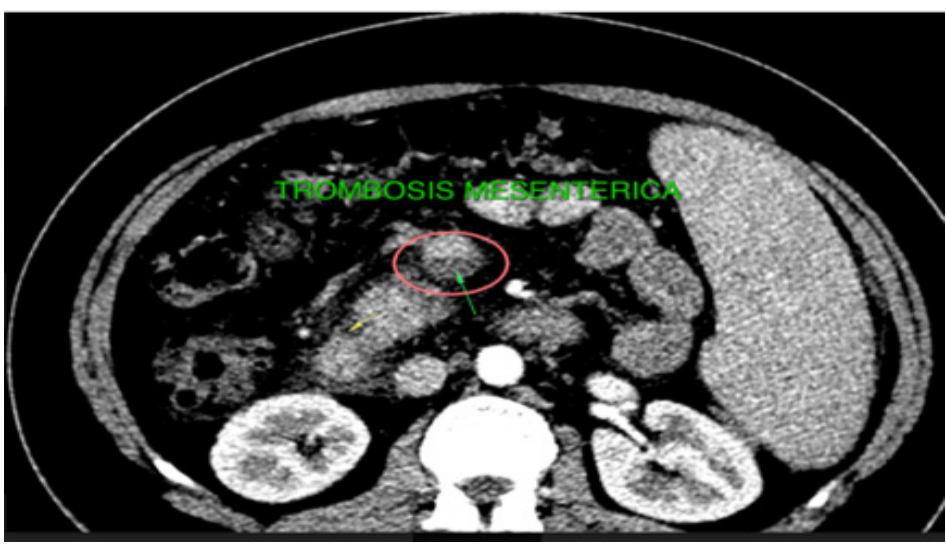

Fig. 3: Corte axial de tomografía de abdomen con inyección de medio de contraste I.V. yodado pone en evidencia defecto de llenado en la mitad posterior de la vena mesentérica superior compatible con trombo mural en dicho segmento venoso. Se agrega al hallazgo esplenomegalia homogénea por la hipertensión portal.

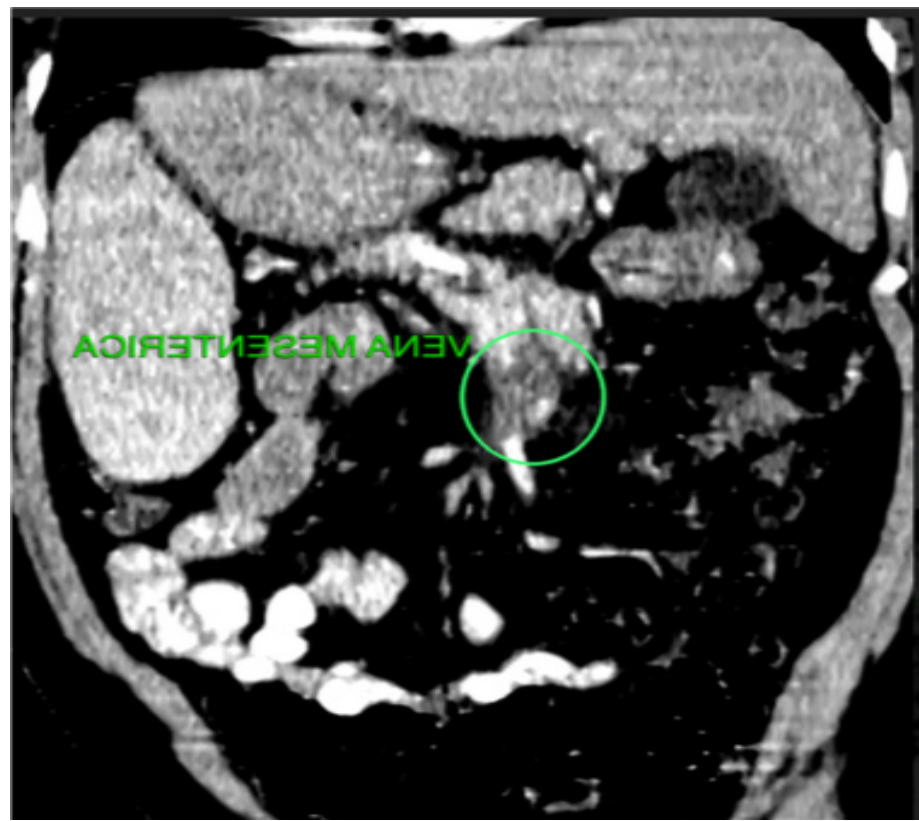

Fig. 4: Corte coronal tomográfico con reformateado MIP del abdomen a nivel de la vena mesentérica superior documentándose defecto de llenado dentro de la vena y ensanchamiento del calibre de la vena debido a la trombosis en el segmento terminal de la misma.

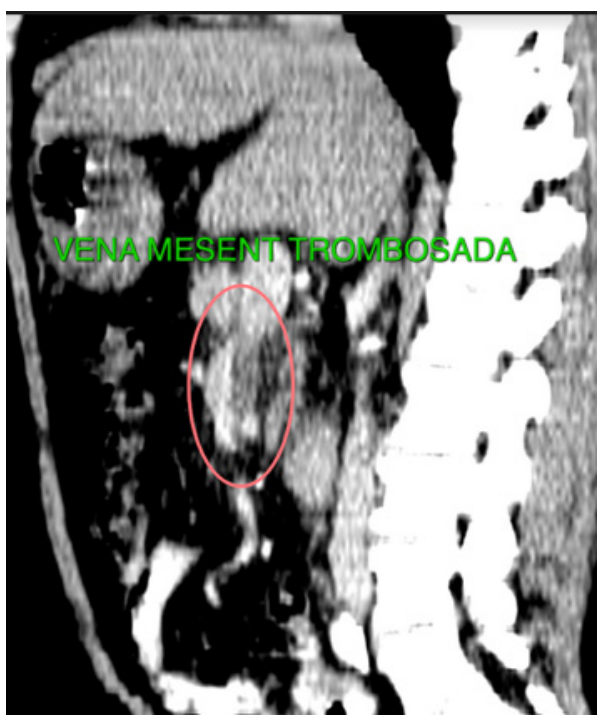

Fig. 5: Corte de tomográfico sagital por reformateado MIP del abdomen a nivel de la vena mesentérica superior viéndose la vena en plano sagital demostrando el defecto de llenado (círculo rojo) hipoatenuado en la mitad posterior del lumen venoso. 
En los estudios radiológicos realizados no se encontró aumento del líquido peritoneal, no había edema de paredes intestinales ni alteración de la grasa mesentérica y el resto del abdomen y pelvis no mostraba alteraciones aparte de los hallazgos de hepatopatía crónica en hígado, la esplenomegalia y circulación hepatofuga escasa. El caso fue notificado al médico tratante y el paciente fue hospitalizado. Se le dio tratamiento anticoagulante teniendo durante su estadía en dos ocasiones melena y sangrado digestivo alto por varices sangrantes vistas con endoscopia por lo que se le realizó ligadura de las mismas en dos ocasiones. Dos semanas después se le realizó de nuevo una tomografía axial computada multicorte hospitalariamente y se demostró ausencia del trombo en la vena mesentérica superior. El paciente no ha tenido datos de sepsis, está estable sin dolor abdominal y en control en el Servicio de Gastroenterología Hospitalario.

\section{Discusión}

La circulación venosa también se puede ver comprometida en la estrangulación del intestino como lo observado en volvulus, intusucepción o en obstrucción en asa cerrada en donde inicialmente hay flujo venoso a baja presión con subsecuente pérdida del flujo arterial con distensión intestinal, fluido con ascitis y edema de la pared intestinal6,7. Trombosis de la vena mesentérica aguda más comúnmente ocurre cuando existen condiciones patológicas como estados de hipercoagubilidad o neoplasias proliferativas causando trombosis de ramas venosas pequeñas con la consecuente reducción drástica del retorno venoso y formación de vasos colaterales ${ }^{13}$.

Lalocalización deltromboesamenudodeterminado por la causa subyacente. Trombosis debida por ejemplo a pancreatitis, usualmente inicia en los troncos gruesos en sitios de compresión y luego progresa a las venas periféricas. Al contrario, si el origen es por ejemplo debido a un trastorno hematológico primero afecta ramas venosas pequeñas y luego progresa a las venas mayores ${ }^{14}$. Varios métodos de imágenes están disponibles para su diagnóstico actualmente, cada cual tiene sus ventajas y desventajas.
El Ultrasonido Convencional de alta resolución fue muy útil en nuestro caso ya que fue con él que se sospechó de la presencia de trombo en la vena mesentérica, se descartó líquido peritoneal, edema de pared intestinal, descarto de trombosis esplenoportal y confirmó por imagen el aspecto de hepatopatía crónica del paciente; sin embargo al aplicarle el Ultrasonido Doppler Color se pudo demostrar que el trombo estaba en la vena mesentérica pero estaba permeable. Sabemos de las limitaciones que tiene el ultrasonido Doppler color por la superposición de gas intestinal y por ser una técnica operador dependiente que necesita radiólogos con amplia experiencia en el manejo de esta técnica, así como limitaciones para visualizar circulación colateral venosa permeable y sobretodo hacia el retroperitoneo.

El diagnóstico por imágenes no invasivas de una trombosis mesentérica en tomografía Axial Computada pone en evidencia un reforzamiento de la pared intestinal que es variable dependiendo de la duración de la obstrucción, al inicio con reforzamiento y solamente con compromiso venoso pero cuando hay ya compromiso arterial aparece disminución del reforzamiento mural en la pared con aspecto hipodenso. La tomografía axial computada tiene una alta sensibilidad de 83 al $100 \%$ y especificidad del $61 \%$ al $93 \%$ para el dignóstico de estrangulación con hallazgos asociados de ascitis adyacente y cambios de densidad en la grasa mesentérica que son los hallazgos más específicos de isquemia intestinal| ${ }^{10,11}$.

Trombosis dentro de la vena mesentérica puede aparecer como un defecto de llenado de baja atenuación en el TAC con medio de contraste I.V. y este hallazgo puede ser visualizado en aproximadamente $90 \%$ de los casos de isquemia intestinal venosa. Además, debido a la obstrucción al flujo venoso es observado típicamente un engrosamiento y edema de las venas mesentéricas ${ }^{12,13}$. 


\section{Table I}

\begin{tabular}{|c|c|c|c|}
\hline $\begin{array}{l}\text { Clinical Risk } \\
\text { Factors }\end{array}$ & $\begin{array}{l}\text { Cardiovascular disease: } \\
\text { Atrial fibrillation, post- } \\
\text { Myocardial infarction, aortic } \\
\text { injury, atherosclerosis. } \\
\text { septic emboli. systemic } \\
\text { vasculitis. }\end{array}$ & $\begin{array}{l}\text { Bowel } \\
\text { strangulation, } \\
\text { hypercoagulable } \\
\text { state, portal } \\
\text { hypertension, } \\
\text { venous trauma, } \\
\text { infection }\end{array}$ & $\begin{array}{l}\text { Hypotension, } \\
\text { heart failure, } \\
\text { recent surgery } \\
\text { or trauma, } \\
\text { medications } \\
\text { including } \\
\text { recreational }\end{array}$ \\
\hline Vasculature & $\begin{array}{l}\text { Arterial filling defect, severe } \\
\text { arterial narrowing, } \\
\text { dissection, aneurysm }\end{array}$ & $\begin{array}{l}\text { Venous filling } \\
\text { defect, often with } \\
\text { enlarged venous } \\
\text { diameter }\end{array}$ & Non-specific \\
\hline $\begin{array}{l}\text { Bowel Wall } \\
\text { Thickness }\end{array}$ & $\begin{array}{l}\text { May be thin acutely, but } \\
\text { may be thickened and } \\
\text { involved with hematoma, } \\
\text { edema, or inflammation }\end{array}$ & $\begin{array}{l}\text { Thickened and } \\
\text { edematous }\end{array}$ & $\begin{array}{l}\text { Generally } \\
\text { thickened }\end{array}$ \\
\hline $\begin{array}{l}\text { Bowel Wall } \\
\text { Enhancement }\end{array}$ & $\begin{array}{l}\text { Variable; diminished or non- } \\
\text { enhancement in regions of } \\
\text { pale ischemia; } \\
\text { hyperenhancement in areas } \\
\text { of reperfusion }\end{array}$ & $\begin{array}{l}\text { Diminished } \\
\text { enhancement of } \\
\text { mucosa and } \\
\text { serosa, target } \\
\text { appearance }\end{array}$ & $\begin{array}{l}\text { Diminished } \\
\text { enhancement }\end{array}$ \\
\hline Mesentery/Fat & $\begin{array}{l}\text { Mesenteric fat stranding } \\
\text { with free fluid associated } \\
\text { with the territory of ischemia }\end{array}$ & $\begin{array}{l}\text { Mesenteric fat } \\
\text { stranding with } \\
\text { free fluid } \\
\text { associated with } \\
\text { the territory of } \\
\text { ischemia }\end{array}$ & $\begin{array}{l}\text { Mesenteric fat } \\
\text { stranding with } \\
\text { free fluid } \\
\text { associated with } \\
\text { the territory of } \\
\text { ischemia }\end{array}$ \\
\hline
\end{tabular}

Tabla 1: Hallazgos radiológicos en CT multidetector multicorte en Trombosis Venosa Mesentérica con inyección de medio de contraste I.V. ${ }^{8,9}$

Fue la tomografía axial computada multicorte con medio de contraste intravenoso que comprobó la presencia de un trombo mural en el segmento terminal de la vena mesentérica superior con un lumen parcialmente permeable. En nuestro caso no fue utilizada la Resonancia Magnética porque estaba claro del diagnóstico y por limitaciones económicas; sin embargo se reconoce el gran aporte que puede hacer la angioresonancia con gadolinio en estos casos. La angiografía digital del sistema arterial y venoso mesentérico está reservado para casos de diagnóstico complicado con las técnicas no invasivas y actualmente se utiliza también para terapia por cateterismo en el manejo de trombosis portal y mesentérica venosa ${ }^{9}$.

\section{Conclusión:}

La triada de un área de baja atenuación en la vena mesentérica superior, engrosamiento de la pared intestinal y la presencia fluido peritoneal ${ }^{10}$, según Michelle $S$ et al 10 sugiere que la laparotomía debe ser realizada por infarto intestinal y comenta que la presencia de líquido peritoneal indica severa isquemia mesentérica secundaria a oclusión venosa esplácnica y por el contrario no recomienda la posibilidad de cirugía si no hay liquido peritoneal.

Los radiólogos juegan un rol crítico en el diagnóstico y apropiado de pacientes con isquemia mesentérica. El ultrasonido convencional y el Doppler Color siguen teniendo un papel importante en pacientes con dolor abdominal y en pacientes con riesgo de trombosis venosa mesentérica realizando un rastreo del abdomen aunque tiene sus limitaciones ya comentadas. EI TAC multicorte con medio de contraste es un invaluable test de imagen cuando se sospecha de isquemia mesentérica y supera todas las demás pruebas médicas a excepción de la angioresonancia pero la tomografía es de escogencia en muchos centros médicos en estos casos porque la resonancia no está disponible en todos los centros médicos, es más costosa y tiene sus limitaciones de acceso al recinto del resonador. Debido a que la isquemia puede presentarse con múltiples y diferentes apariencias radiológicas entender la vasculatura intestinal y la anatomía mesentérica ayuda a mejorar el diagnóstico de esa desafiante enfermedad.

Se revisa uncaso de una trombosis venosa parcial, incompleta del segmento terminal de la vena mesentérica superior presentando clínicamente síntomas vagos de dolor abdominal pero padeciendo hipertensión portal como factor de riesgo y se utilizaron las técnicas de imágenes disponibles en este momento las cuales jugaron un rol importante en detectar el riesgo en camino de una obstrucción completa al evolucionar con el tiempo si no se hubiera hecho el hallazgo. Se hace una revisión de los hallazgos tomográficos 
que se mencionan en la literatura en los casos de isquemia mesentérica la cual está anotada en la tabla 1.

No encontramos en la literatura referencia de casos que describan trombosis venosa parcial de la vena mesentérica o suboclusiva diagnosticada con imágenes médicas por tal motivo lo publicamos debido a que por lo revisado y lo catastrófico del pronóstico de la enfermedad se deben hacer los mayores esfuerzos para pensar en esta entidad en pacientes con dolor abdominal y poseedores de factores de riesgo y así explotar más las posibilidades diagnósticas que pueden brindar la Tomografía Axial Computada y el ultrasonido convencional y el ultrasonido Doppler a color. 


\section{Referencias}

1.Cartwright SL, Knudson MP. (Abril, 2008). Evaluation of Acute Abdominal Pain in Adults. Am Fam Physician. 77(7) 971. Recuperado de https://www.aafp.org/afp/2008/0401/p971.html

2.Ruotolo RA, Evans SR. (Agosto, 1999). Mesenteric ischemia in the elderly. Clinics in Geriatric Medicine. 15(3). Recuperado de https://www.ncbi.nlm.nih.gov/pubmed/10393740

3.https://www.sciencedirect.com/science/article/abs/pii/S0749069018300478

4.Wiesner W, Mortele KJ, Glickman JN, et al. (Diciembreer,2001). Pneumatosis intestinalis and portomesenteric venous gas in intestinal ischemia; Correlation of CT findings with severity of ischemia and clinical outcome. AJR Am J Roentgenol. 177(6). Recuperado de https://www.ajronline.org/doi/full/10.2214/ajr.177.6.1771319

5.Levine JS, Jacobson ED. (Noviembre, 2008) Intestinal ischemic disorders. 13(1). Recuperado de https://www.karger.com/Article/ Abstract/171483

6.Brandt L, Boley S, Golbberg L, et al. (Setiempre, 1981). Colitis in the elderly. A reappraisal. Am J Gastroenterol. 76(3). Recuperdado de https://www.ncbi.nlm.nih.gov/pubmed/9356638

7.Rha SE, Ha HK, Lee SH, et al. (Enero, 2000). CT and MR imaging findings of bowel ischemia from various primary causes.

Radiographics;20(1):29-42. https://doi.org/10.1148/radiographics.20.1.g00ja0629

8.Turnage RH, Guice KS, Oldham KT. (Junio, 1994). Endotoxemia and remote organ injury following intestinal reperfusion. J Surg Res. 56(6). Recuperado de https://www.journalofsurgicalresearch.com/article/S0022-4804(84)71091-2/pdf

9. Harpreet S. Dhatt, Md, Spencer C. Behr, MD, Aaron Miracle, MD, Zhen Jane Wang, MD, Benjamin M, Yeh, MD. (Noviembre, 2015). Radiological evaluation of bowel ischemia. Radiol Clin North Am. 53(6). Recuperado de https://www.ncbi.nlm.nih.gov/pmc/articles/ PMC4633709/

10.Michelle S. Bradburry, MD, PhD. Peter V. Kavanagh, MD. Robert E.Bechtold, MD. et al. (May, 2002). Mesenteric Venous Thrombosis: Diagnosis and Noninvasive Imaging. Radiographics 22(3), 527-541. https://doi.org/10.1148/ radiographics.22.3.g02ma10527

11.Balthazar EJ, Liebeskind ME, Macari M. (Noviembre, 1997). Intestinal ischemia in patients in whom small bowel obstruction is suspected: evaluation of accuracy, limitations, and clinical implications of CT in diagnosis. Radiology 205(2):519-22. https://doi. org/10.1148/radiology.205.2.9356638

12.Zalcman M. Sy M, Donckier V, et al. (Diciembre, 2000). Helical CT signs in the diagnosis of intestinal ischemia in small-bowel obstruction. AJR Am J Roentgenol. 175(6). Recuperado de https://www.ajronline.org/doi/full/10.2214/ajr.175.6.1751601

13.Bradbury MS, Kavanagh PV, Bechtold RE, et al. (May, 2002). Mesenteric venous thrombosis: diagnosis and noninvasive imaging. Radiographics. 175(6). https://doi.org/10.1148/radiographics.22.3.g02ma10527

14. Harward TR, Green D, Bergan JJ, et al. (Junio, 1998). Mesenteric venous thrombosis. J Vasc Surg. 9(2). Recuperado de https:// www.jvascsurg.org/article/0741-5214(89)90053-0/fulltext

15.Rafael Duran, MD, Alban L. Denys, MD. Igor Letovanec, MD. Reto A. Meuli, PhD, MD, Sabine Schmidt, MD. (Febrero, 2012) Multidetector CT Features of Mesenteric Vein Trombosis. Radiographics. 32(5). Recuperado de https://pubs.rsna.org/doi/pdf/10.1148/ $\operatorname{rg} .325115100$

16.Kumar S, Sarr MG, Kamath PS. (Diciembre, 2001). Mesenteric venous thrombosis. N Engl J Med. 345(23):1683-1688. https://doi. org/10.1056/NEJMra010076

17.Freeny PC, Marks WM. (Setiembre,1986). Patterns of contrast enhancement of benign and malignant hepatic neoplsms during bolus dynamic and delayed CT. Radiology. 160(3). https://doi.org/10.1148/radiology.160.3.3016794 